\title{
Characterization of a novel developmentally retarded mutant (drm1) associated with the autonomous flowering pathway in Arabidopsis
}

\author{
Yong ZHU ${ }^{1,2, *}$, Hui Fang ZHAO ${ }^{1,3, *}$, Guo Dong REN ${ }^{1}$, Xiao Fei YU ${ }^{1}$, Shu Qing CAO ${ }^{1}$, Ben Ke KUAI ${ }^{1,4, * *}$ \\ ${ }^{1}$ Department of Biochemistry, Ministry of Education Key laboratory for Biodiversity Science and Ecological Engineering, \\ School of Life Sciences, Fudan University, 220 Handan Road, Shanghai 200433, China; \\ ${ }^{2}$ School of Horticulture and Gardening, Southwest Forestry College, Kun Ming 650224, China; \\ ${ }^{3}$ Department of biology, School of Life and Environment Science, Shanghai Normal University, Shanghai 200234, China \\ ${ }^{4}$ Fudan Centre for Genetic Diversity and designed Agriculture, Fudan University, 220 Handan Road, Shanghai 200433, \\ China
}

\begin{abstract}
A developmentally retarded mutant ( $\mathrm{drm} 1$ ) was identified from ethyl methanesulfonate (EMS)-mutagenized M2 seeds in Columbia (Col-0) genetic background. The $d r m 1$ flowers $109 \mathrm{~d}$ after sowing, with a whole life cycle of about $160 \mathrm{~d}$. It also shows a pleiotropic phenotype, e.g., slow germination and lower germination rate, lower growth rate, curling leaves and abnormal floral organs. The $d r m 1$ mutation was a single recessive nuclear mutation, which was mapped to the bottom of chromosome 5 and located within a region of 20-30 kb around MXK3.1. There have been no mutants with similar phenotypes reported in the literature, suggesting that $D R M 1$ is a novel flowering promoting locus. The findings that the $\mathrm{drm} 1$ flowered lately under all photoperiod conditions and its late flowering phenotype was significantly restored by vernalization treatment suggest that the $d r m 1$ is a typical late flowering mutant and most likely associated with the autonomous flowering pathway. The conclusion was further confirmed by the revelation that the transcript level of $F L C$ was constantly upregulated in the $d r m 1$ at all the developmental phases examined, except for a very early stage. Moreover, the transcript levels of two other important repressors, EMF and TFL1, were also upregulated in the $d r m 1$, implying that the two repressors, along with $F L C$, seems to act in parallel pathways in the $d r m 1$ to regulate flowering as well as other aspects of floral development in a negatively additive way. This helps to explain why the $d r m 1$ exhibits a much more severe late-flowering phenotype than most late-flowering mutants reported. It also implies that the $D R M 1$ might act upstream of these repressors.
\end{abstract}

Keywords: $d r m 1$, late-flowering, autonomous flowering pathway, Arabidopsis thaliana.

\section{INTRODUCTION}

The transition from vegetative growth to reproduction is one of the most important developmental events in flowering plants since it is related to the competence and survivability of a particular species living in a particular environment. The flowering time, as the phenotypic indicator of this transition, is either induced by environmental factors or regulated by endogenous signals. Four major pathways controlling flowering time have been defined in Arabidopsis [1-4].

\footnotetext{
*These authors contributed equally to this work

**Correspondence: Ben Ke KUAI

Tel: 86-21-65643676, Fax: 86-21-65642648;

E-mail: bkkuai@fudan.edu.cn
}

The photoperiod flowering pathway regulates flowering time by responding to long-day conditions. Late-flowering mutants in this pathway flower late under long-day conditions but similarly or even identically to the wild type under short days. These mutants are weakly, or not at all, sensitive to vernalization. CO, CRY2, FHA, GI, FT and $F W A$ are some components characteristic in this pathway [5]. Regulation of flowering time in response to seasonal day length fluctuations is mediated by the interactions between light signals and intrinsic time-keeping mechanisms that are associated with the circadian clock [6, 7]. CRY2 and $P H Y A$, candidates for photoreceptors that perceive the photoperiod under long days, entrain the circadian clock to oscillate within a period of $24 \mathrm{~h}[8,9]$. The components of the oscillator include TOCl, CCA1 and $L H Y$ genes, which 
are also implicated in the photoperiodic induction of flowering $[10,11]$.

The Gibberellin (GA) promotes flowering and is absolutely required for flowering in non-inductive short days. Mutations in genes involved in GA biosynthesis and signaling result in delayed flowering. A growing number of genes associated with this pathway have been identified, including SPY, PHOR1, RGLs, RGA, GAI, GA4, GA5 and $F P F 1[2,12]$.

The vernalization responsive pathway regulates flowering in response to extended exposure to cold temperature (vernalization). Many naturally occurring mutants flower very late but flower early if exposed to low temperatures for 4 to $8 \mathrm{w}$. The vernalization response is mediated by dominant alleles of two genes, FRI and FLC, through reducing the expression of $F L C[13,14]$. Other genes involved in vernalization response include $V R N 1$ and VRN2 [15, 16], HOS1 [17] and VIP1-7 [18].

The autonomous pathway is defined by one group of late flowering mutants, such as $f c a, f p a, f v e, f l d, l d$, and $f y$, which flower late under both long-day and short-day conditions. However, the late flowering phenotypes of these mutants can be overcome by vernalization or exposure to far red-enriched light [5]. The characteristic feature of these mutants is that they all contain much higher levels of $F L C$ transcript than the wild-type plant or late-flowering mutants associated with the photoperiod flowering pathway or the GA pathway $[13,19,20]$.

Different flowering time pathways are known to interconnect and converge on the activation of the same flowering-time genes, which are termed as the flowering-time pathway integrators. Thus far, three genes have been identified: FT, SOC1 (AGL20) and $L F Y$ [21-23]. These floral integrators then activate the expression of the downstream floral organ identity genes, $A P 3, P I$ and $A G$, leading to the development of floral organs [24-27].

A large number of genes, functioning as floral repressors, have also been identified from early flowering mutants, including EMF1 and 2, TFL1 and 2, CLF,EBS1, EFS, ELF3, ELF4 ELF5, ESD4, FIE, SYD,SVP and TOE [28-31]. These floral repressor genes interconnect with the network of flowering pathways in one way or another to regulate flowering negatively. For example, ESD4 is involved in the autonomous floral promotion pathway [29, 32] and SVP interacts with the photoperiod pathway [33]. Moreover, some of the repressor genes, such as $E M F$ and $T F L$, also affect the development of inflorescences and floral organs in Arabidopsis [34-36].

In this study, a developmentally retarded mutant ( $\mathrm{drm} 1)$ was isolated in Arabidopsis, which flowers extremely late and shows a pleiotropic phenotype. DRMI was located within a region of $20-30 \mathrm{~kb}$ around MXK3.1 on the bottom of chromosome 5 and appears to be a novel flowering promoting locus. It was further defined as a novel component of the autonomous flowering pathway. Repressors $F L C, E M F$ and $T F L 1$ were also found to be involved in the regulation of the severe phenotype of late flowering as well as floral abnormality in the drm 1 .

\section{MATERIALS AND METHODS}

\section{Plant materials and growth conditions}

All the wild-type and mutant Arabidopsis lines used in this study are in a Columbia (Col-0) background unless indicated otherwise. When grown in soil pots, seeds were sown in square pots $(10 \mathrm{~cm}$ in length) with soil [ $\mathrm{v}$ (peat soil): $\mathrm{v}$ (vermiculite): $\mathrm{v}$ (pearlite) $=3: 9: 0.5$, Shanghai Institute of Landscape Science] presoaked with PNS medium. Plants were grown in a controlled room with $22 \pm 2{ }^{\circ} \mathrm{C}$ temperature and $\sim 100 \mu \mathrm{mol} \mathrm{m}-2 \mathrm{~s}-1$ light intensity under standard long-day conditions (16 h light $/ 8 \mathrm{~h}$ dark).

For photoperiod treatment, plants on soil pots were grown under various photoperiod conditions, including $24 \mathrm{~h}$ continuous light, $16 \mathrm{~h} \mathrm{light} / 8 \mathrm{~h}$ dark, $8 \mathrm{~h}$ light/ $16 \mathrm{~h}$ dark and $4 \mathrm{~h} \mathrm{light} / 20 \mathrm{~h}$ dark.

For vernalization treatment, imbibed seeds were placed at $4^{\circ} \mathrm{C}$ in the dark for $30 \mathrm{~d}$ before they were transferred to long-day conditions, as described above, whereas untreated seeds were kept at room temperature for $28 \mathrm{~d}$ and then sowed on pots and placed at $4{ }^{\circ} \mathrm{C}$ in the dark for $2 \mathrm{~d}$ before being transferred to long-day conditions.

\section{Isolation and characterization of the $\mathrm{drm} 1$ mutant}

Ethyl methanesulfonate (EMS) mutagenized M2 seeds in Col-0 genetic background were purchased from Lehle Seeds (Round Rock, TX, USA). For mutant screening, M2 seeds were sown on watersoaked soil, and treated at $4^{\circ} \mathrm{C}$ for $2 \mathrm{~d}$ before being transferred to the growth room. The $d r m 1$ mutant was isolated from M2 population for its abnormal phenotype. The phenotype of the $d r m 1$ was stable and reproducible in M3 and onward generations under the growth conditions described above.

The drm 1 plants were backcrossed with the wild type for 3 times, and the resulting homozygous $d r m 1$ plants were used for all the analysis. The phenotype of the $d r m 1$ mutant was characterized under long-day photoperiod. Bolting time was measured as days from seed sowing to the first flower bud emerging while flowering time was recorded to the first flower opening. Rosette leaves were counted when a visible inflorescence of $\sim 3 \mathrm{~cm}$ was apparent.

\section{Genetic analysis and mapping of DRM1 locus}

For genetic analysis, $d r m 1$ plants were crossed with wild-type plants reciprocally, and the resulting F1 seedlings were allowed to self-pollinate to produce $\mathrm{F} 2$ populations. The F1 and F2 seedlings were scored for either mutant or wild phenotype.

For mutation locus mapping, homozygous $d r m 1$ plants were crossed to wild-type plants in Landsberg background. From the segregating F2 population, 2300 homozygous drm 1 plants were selected to make a mapping population, and DNA was extracted from each of these plants. The linkage between the mutation locus and molecular markers was determined by using simple sequence length polymorphism (SSLP) markers [37]. For fine mapping, we designed a set of novel SSLP markers by using the Cereon arabidopsis polymorphism collection (Tab. 1). Mapping procedure was performed as described in Lukowitz and Jander [38, 39]. All the primers were 
Tab. 1 Newly designed SSLP primer sets

\begin{tabular}{|c|c|c|c|c|c|c|c|}
\hline \multirow[t]{2}{*}{ Marker } & \multirow[t]{2}{*}{ BAC I } & \multirow{2}{*}{\multicolumn{2}{|c|}{ Position in $\mathrm{BAC}$}} & \multicolumn{2}{|c|}{ Oligonucleotide sequences } & \multicolumn{2}{|c|}{$\begin{array}{l}\text { PCR product } \\
\text { size(bp) }\end{array}$} \\
\hline & & & & Forward primer & Reverse primer & Ler & Col-0 \\
\hline AtMAC9.1 & MAC9 & 21587 & 21748 & 5'TGCTTCCCTACACTGCTC3' & 5'GTTCCGCTTCTTCTCCTG3' & 136 & 162 \\
\hline AtMBK5.1 & MBK5 & 30767 & 30786 & 5'ATCACTGTTGTTTACCATTA3' & 5'GAGCATTTCACAGAGACG3' & 180 & 207 \\
\hline AtT12B11.1 & T12B11 & 5348 & 5497 & 5'GAAGACTGTTGTGATGAAATGG & 3'CGTCACAGAACCGTCACATA3' & 167 & 150 \\
\hline AtMVP7.1 & MVP7 & 20187 & 20339 & 5'TTGGACCGACTCTCATTA3' & 5'CAAATACATCCACAGGGG3' & 169 & 153 \\
\hline AtMXK3 .2 & MXK3 & 13130 & 13233 & 5'TCGCAAAATCAAAATCTT3' & 5'ATCAACTAAAATAGCGGA3' & 98 & 104 \\
\hline AtMXK3 .1 & MXK3 & 71580 & 71724 & 5'GATACAGTCCGATGATGTCCTAAT3' & ' 5'AGACCAAACCCAAAAAACAAAC3' & 159 & 145 \\
\hline AtMNA5.1 & MNA5 & 76414 & 76577 & 5'CAGGTGGTAAGGGAAGTAAACA3' & 5'GCTTAGGCTTTCTCATCCATTG3' & 147 & 164 \\
\hline AtF1505.1 & F1505 & 11766 & 11949 & 5'AAGTAACCACCATCACCAACGA3' & 5'CTGTCTCCGAAGGTAACAATAA3' & 168 & 184 \\
\hline AtMQN23.1 & MQN18 & 867403 & 67602 & 5'GTTGACTGGATTTGTTTGGT3' & 5'ATCGTTGATTGGTCTCTCTA3' & 182 & 200 \\
\hline
\end{tabular}

synthesized by TaKaRa Biotechnology Co.

\section{Total RNA extraction and semi-quantitative RT-PCR}

Total RNA was extracted from mixed rosette leaves of wildtype as well as $d r m 1$ plants using the TRI reagent (Invitrogen). First-stand cDNA synthesis was performed with $3 \mu \mathrm{g}$ total RNA using a SuperScript kit (Gibco BRL), and the products were standardized for semi-quantitative RT-PCR using $\beta$-actin 11 as a control.

The semi-quantitative RT-PCR was adopted to monitor the change in transcript levels of flowering time genes. Gene-specific primers and the amplification cycles used were as for each gene are as supplied in Supplemental Tab. 1. PCR conditions were as follows: $5 \mathrm{~min}$ at $94^{\circ} \mathrm{C}$, then $20-40$ cycles of $94^{\circ} \mathrm{C}$ for $45 \mathrm{sec}, 55^{\circ} \mathrm{C}$ for $90 \mathrm{sec}, 72^{\circ} \mathrm{C}$ for $45 \mathrm{sec}$, and then $72^{\circ} \mathrm{C}$ for $5 \mathrm{~min}$. The amplified fragments were separated on a $1.2 \%$ agarose gel. Reproducible expression patterns were obtained for each of the genes with RNA samples extracted from different batches of seedlings.

\section{RESULTS}

\section{Isolation and phenotypic characterization of the drm 1 mutant}

In a screening for stay-green mutants using a dark stressed approach, a number of developmentally retarded and / or late-flowering mutants were obtained from ethyl methane sulphonate (EMS) mutagenized M2 seeds of Columbia (Col) ecotype of Arabidopsis thaliana. Of them, an extremely slow-developing mutant, designated $\mathrm{drm} 1$ (developmentally retarded mutant) was selected for further analysis.

Under long-day conditions, all the developmental phases of the $\mathrm{drm} 1$ mutant are severely retarded, and a period of $109 \mathrm{~d}$ is needed from sowing to flowering compared to 42 $\mathrm{d}$ for the wild type. Consequently, the plant size of the mutant is much smaller at the early stage of development, but becomes comparable to that of the wild type towards flowering time. In fact it can produce as many as 58 rosette leaves compared to 24 of the wild-type at the time of bolting due to the extended period of vegetative growth. Besides, the process of senescence at plant level is also significantly delayed, and therefore an extremely long lifespan was recorded (Tab. 2 and Fig. 1).

The mutation displays a pleiotropic phenotype. Its germination rate is significantly lower and its germination speed is 3-4 $\mathrm{d}$ slower compared to the wild-type (Tab. 2). Its rosette leaves, thick and pale green at the seedling stage

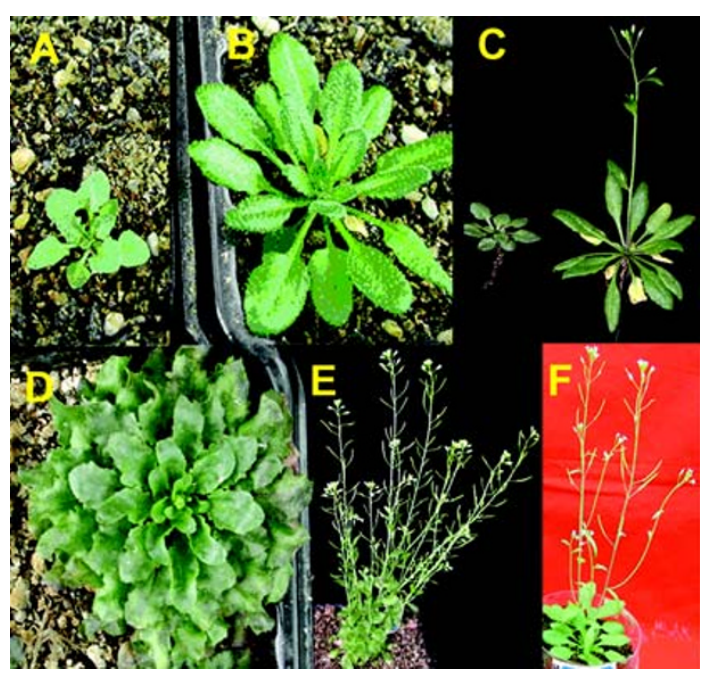

Fig. 1 Phenotype of the $d r m 1$. (A) A plantlet of the $d r m 1$ and (B) a plant of the wild-type grown under long-day conditions in soil supplemented with PNS medium $40 \mathrm{~d}$ after sowing. (C) A plant of the $\mathrm{drm} 1$ (left) and a plant of the wild-type (right) $48 \mathrm{~d}$ after sowing. (D) A plant of the $d r m 1$ with a visible flower bud and increased number of rosette leaves $90 \mathrm{~d}$ after sowing. (E) A plant of the $\mathrm{drm} 1$ (124 d-old) showing more multiple inflorescences compared to those of the $(\mathbf{F})$ wild-type (60 d-old) at the same developmental phase. 
(Fig. 1A), curl up after fully-grown (Fig. 1D). Furthermore, the $d r m 1$ produces significantly more inflorescences, particularly more secondary, tertiary and quaternary ones (Fig. 1E, F; Fig. 2E). Although the inflorescences of the drm 1 bear more flowers, the fertilities of many flowers are severely reduced due to unusual shorter stamens, which result in a number of infertile siliques and / or seeds (Fig. 2A, B). Abnormal numbers of petals (5 or 6 ) and stamens ( 5 or 3 ) were also observed (Fig. 2 C, D).

To analyze the inheritance of the $d r m 1$ mutation, crosses between $d r m 1$ plants and wild-type plants were made reciprocally. No mutant phenotype was observed in the resulting F1 plants and a $1(\mathrm{drm} /)$ to 3 (WT) segregation ratio was revealed in all $\mathrm{F} 2$ populations. Considering the differential germination rates between the drml and the wild type, $\chi^{2}$ test was conducted again based on the converted data and no substantial inconsistency was found between the two calculations (Tab. 3). These results strongly suggest that the mutant phenotype was caused

Tab. 2 Developmental characteristics of the $d r m 1$

\begin{tabular}{lcc}
\hline Growth and developmental parameters & The wild-type & $d r m 1$ \\
\hline Days needed for Seed Germination & $3 \pm 0.2 \mathrm{a}$ & $7 \pm 0.5 \mathrm{a}$ \\
Rate of Seed Germination (\%) & 95 & 86.2 \\
Numbers of Rosette Leaves when Bolting & $24 \pm 2.0 \mathrm{a}$ & $58 \pm 4.2 \mathrm{a}$ \\
Days for bolting & $42 \pm 1.6 \mathrm{a}$ & $88 \pm 3.8 \mathrm{a}$ \\
Days for opening of First Flower & $46 \pm 1.8 \mathrm{a}$ & $109 \pm 3.6 \mathrm{a}$ \\
Days for formation of Fifth Silique & $52 \pm 2.2 \mathrm{a}$ & $116 \pm 3.6 \mathrm{a}$ \\
Days for death of Last Rosette leaf & $75 \pm 3.2 \mathrm{a}$ & $145 \pm 4.2 \mathrm{a}$ \\
Days for death of Last cauline leaf & $82 \pm 3.1 \mathrm{a}$ & $157 \pm 4.5 \mathrm{a}$ \\
\hline
\end{tabular}

a, Means of 20 observations \pm SE.

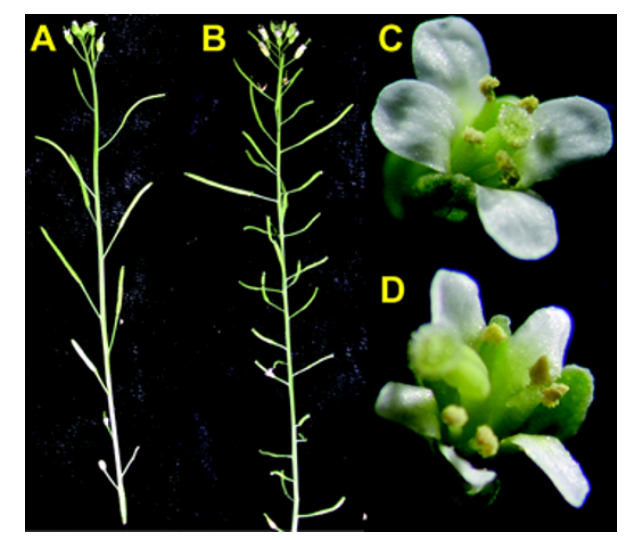

by a single, recessive nuclear mutation.

\section{Mapping of the $D R M 1$ locus}

The DRM1 locus was mapped to the bottom of chromosome 5, flanked by the MBK5 (121.65cM) and MQN $23.1(127.31 \mathrm{cM})$, using SSLP markers [37]. New SSLP markers were designed within this region for fine mapping using a mapping population of 2300 plants. The locus was further located within an interval of $80 \mathrm{~kb}$ between MXK3.2 and F1505.1 markers. As no recombinants were detected by using MXK3.1 marker, we speculated that the chromosomal location of the DRM1 should be within a region of 20-30 $\mathrm{kb}$ around MXK3.1. Sequencing the genomic DNA within this region is under way (Fig. 3).

\section{Physiological and molecular characterization of the late flowering mutant phenotype}

As the late flowering is the most obvious characteristics of the drm 1, experiments were designed to characterize

Tab. 3 F1 phenotypes and F2 segregation ratios of reciprocal crosses

\begin{tabular}{ccccc}
\hline \multirow{2}{*}{ Cross } & Progeny & \multicolumn{2}{c}{ Observed phenotype } & \multirow{2}{*}{$\chi^{2}$ test $(3: 1)$} \\
\cline { 3 - 4 } & & The Wild type $d r m 1$ & \\
\hline \multirow{2}{*}{ Col X drm1 } & F1 & 84 & 0 & \\
& F2 & $3073(3226.7)^{*}$ & $971(1105.0)^{*}$ & $2.11(0.60) \leq 0.10^{*}$ \\
& F1 & 80 & 0 & \\
& F2 & $1314(1379.7)^{*}$ & $420(478.0)^{*}$ & $1.68(0.53) \leq 0.10^{*}$ \\
\hline
\end{tabular}

* Data in bracket were converted according to the differential germination rates.
E

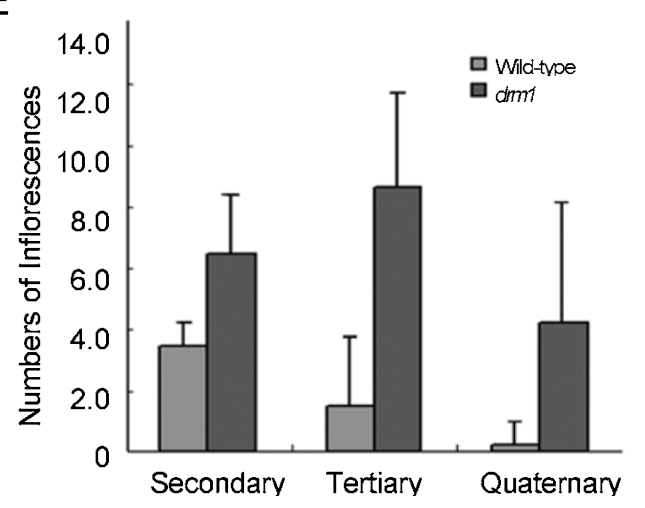

Fig. 2 Floral and inflorescence characteristics of the drm1. (B) A primary inflorescence of the drm 1 showing aberrantly developed siliques in comparison with that of the (A) wild-type. (D) A flower of the $d r m 1$ showing altered number of petals and abnormally shorter stamens compared to those of the (C) wild-type. (E) A graph displaying increased numbers of inflorescences of the $d r m 1$ at different hierarchical levels. 


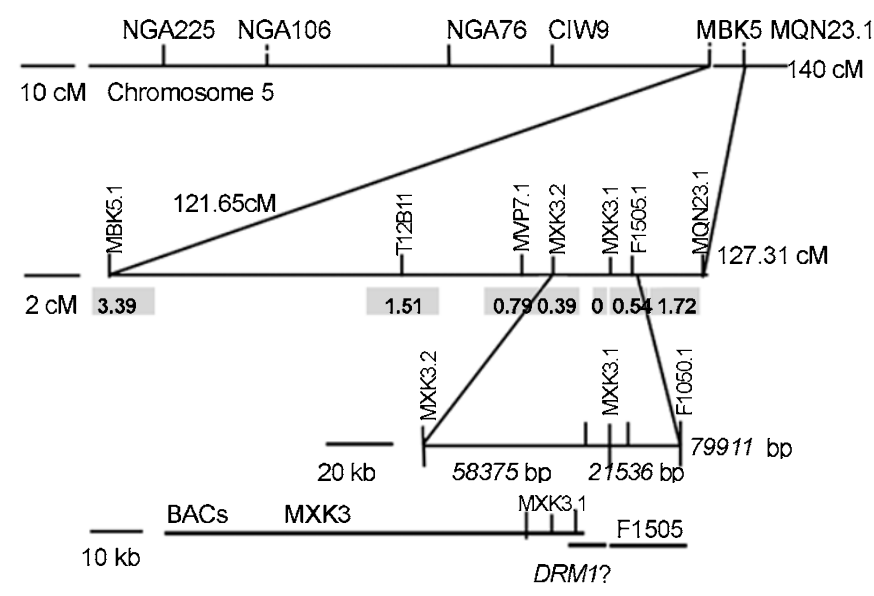

Fig. 3 Mapping of the DRM1 locus. Numbers in bold and shading indicate the rates of recombinants. Numbers in italic indicate physical distances (bp) between molecular markers.

A

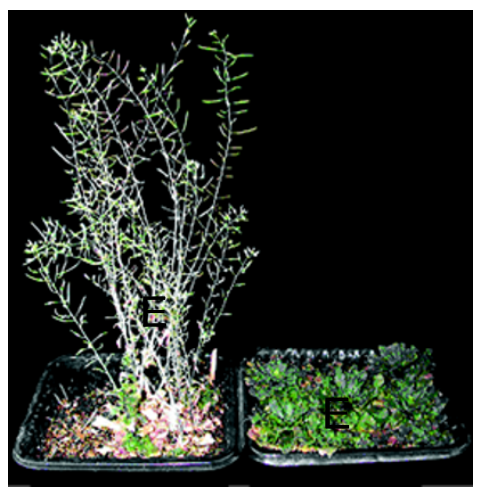

B

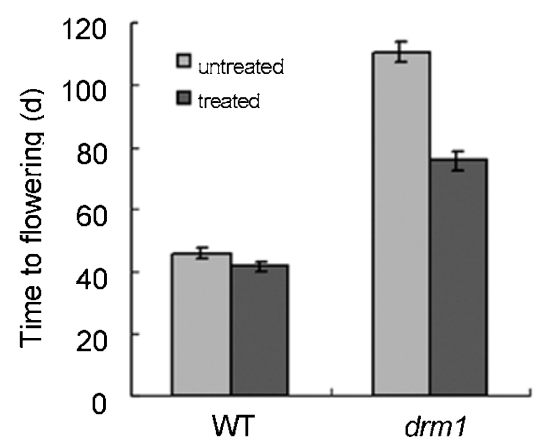

Fig. 4 Effect of vernalization treatment on the flowering time of the $d r m 1$. (A) The late flowering phenotype of the $d r m 1$ was significantly restored (left) by a $30 \mathrm{~d}$ vernalization treatment compared to that of untreated (right), both of which were grown under long-day conditions for $100 \mathrm{~d}$. (B) A graph showing the effect of vernalization treatment on the flowering time of both the $d r m 1$ and the wild-type (WT).

the late flowering mutant phenotype physiologically and molecularly.

Vernalization treatments were carried out as described in Materials and methods. A 30-d vernalization treatment significantly reduced the time needed for flowering in the drm 1 mutant. Treated $d r m 1$ plants flowered on $75.7 \mathrm{~d}$ averagely whereas untreated flowered on $110.5 \mathrm{~d}$ after being transferred to normal long-day conditions from $4^{\circ} \mathrm{C}$. Although not fully restored, the late flowering phenotype of the $d r m 1$ was significantly overcome by the vernalization treatment (Fig. 4).

Responses of the $d r m 1$ to different photoperiods were also determined in order to further define its late flowering phenotype. Although the wild-type always flowered earlier than the $d r m 1$ under all the photoperiods examined, both the $\mathrm{drm} 1$ and the wild-type exhibited photoperiod responses and delayed flowering as the period of illumination was shortened. The $d r m 1$ flowered 98.9 and $295.6 \mathrm{~d}$ after sowing under the photoperiods of $24 \mathrm{~h}$ continuous illumination and $4 \mathrm{~h}$ illumination/20 h dark, respectively (Fig. 5).

To understand the molecular mechanism underlying the late flowering phenotype of the $d r m 1$, transcript levels of some important genes known to play important roles in flowering pathways were examined using the semi-quantitative RT-PCR. It was found that the transcript levels of all the examined integrator genes, $S O C 1(A G L 20), F T$ and $L F Y$, were significantly down-regulated in the $d r m 1$ compared with those in the wild-type at the similar phase of vegetative growth, whereas those of the flowering repressor genes, FLC,EMF1,EMF2 and TFL1, were significantly up-regulated (Fig. 6). The transcript levels of the above repressor genes were all found to be markedly up-regulated at all examined phases (vegetative, transitional and flowering) in the $d r m 1$ compared with those in the wildtype, except that that of the $F L C$ was unexpectedly detected to be down-regulated in the $20 \mathrm{~d}$-old $\mathrm{drml}$ (Fig. 7). These results strongly suggest that the DRM1 mutation

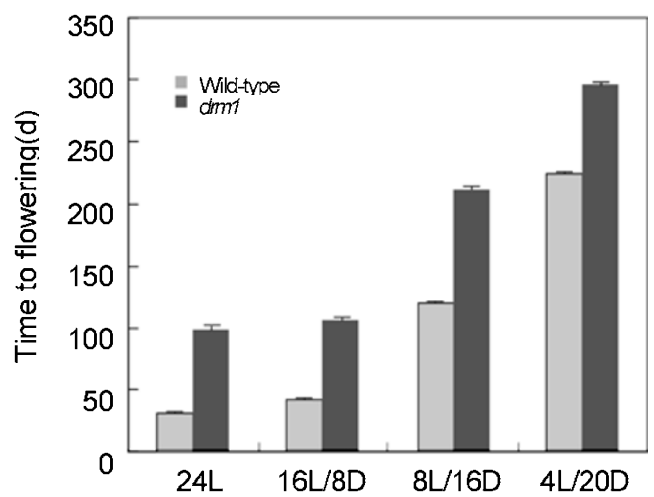

Fig. 5 Effects of photoperiod treatments on the flowering time of both the $d r m 1$ and the wild-type. 24L, $24 \mathrm{~h}$ continuous illumination; $16 \mathrm{~L} / 8 \mathrm{D}, 16 \mathrm{~h}$ illumination / $8 \mathrm{~h}$ darkness; $8 \mathrm{~L} / 16 \mathrm{D}, 8 \mathrm{~h}$ illumination / $16 \mathrm{~h}$ darkness; 4L/20D, $4 \mathrm{~h}$ illumination / $20 \mathrm{~h}$ darkness. 


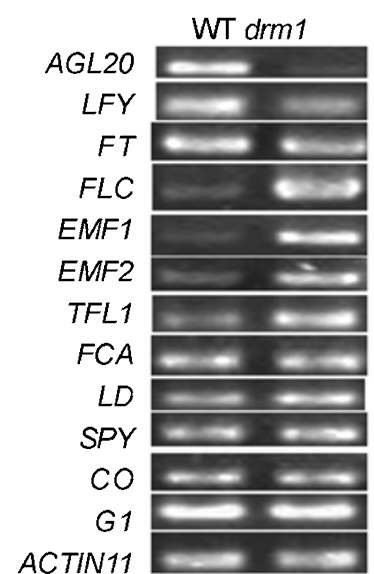

Fig. 6 Transcript levels of flowering-related genes detected in the drm 1 and the wild-type (WT) at a similar vegetative phase (75 d after sowing for the $d r m 1$ and $35 \mathrm{~d}$ for the wild-type) using the semiquantitative RT-PCR.

do affect the expression of flowering genes and the $d r m 1$ can therefore be considered as a late flowering mutant. Considering the finding that a significant up-regulation of $F L C$ expression was detected in most developmental phases, it was postulated that the mutation was most likely associated with the autonomous flowering pathway. This postulation is in accordance with the finding that no significant differences were detected in the transcript levels of other examined flowering genes, such as $G I, C O$ (components of photoperiod flowering pathway), SPY (a component of the GA flowering pathway), $F C A$ and $L D$ (components of the autonomous flowering pathway upstream of $F L C$ ), which are all characteristic components of other flowering pathways (Fig. 6).

\section{DISCUSSION}

The phenotype of the $d r m 1$ is comparable to those of extended lifespan mutants in other model organisms

The flowering time is an important characteristic of a species in plants, and flowering pathways guaranteeing flowering at the right time under particular conditions have been identified both intensively and extensively in Arabidopsis thalinia. Mutations in genes involved in these flowering pathways result in either early flowering or late flowering [1]. The flowering time in plants is a trait considered to be equivalent to the lifespan in animals, and a list of lifespan extending or shortening mutants resulted from the mutations of a conserved pathway have also been observed in other model organisms [40]. Interestingly, the $d r m 1$, along with a large number of other late flowering mutants, shares a similar pleiotropic phenotype with most lifespan extending animal mutants, such as reduced

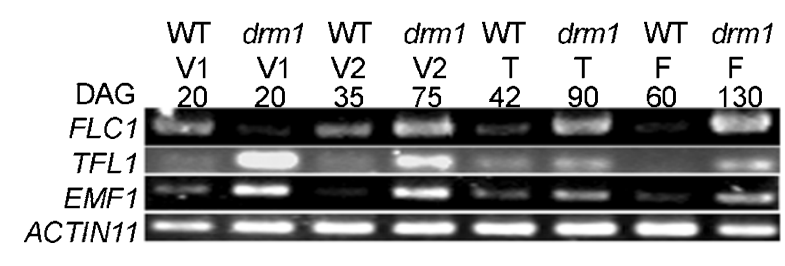

Fig. 7 Transcript levels of flowering repressor genes detected in the drm 1 and the wild-type (WT) at different developmental phases using the semi-quantitative RT-PCR . V: vegetative growth phase; T: transitional phase (floral bud just visible by naked eye); F: flowering phase.

fertilities, small sizes and lower growth rates, indicating that these crucial life processes are evolutionarily linked through certain signal pathways [40].

\section{The DRM1 is likely a novel flowering promoting locus} involved in the autonomous flowering pathway

To our best knowledge, no mutants with a similar phenotype to that of the drm 1 have been reported in the literature. According to the rates of recombinants, the $D R M 1$ mutation is located to an interval of $79.9 \mathrm{~kb}$ between MXK3.2 and F1505.1 markers on the bottom of chromosome 5. So far, no known genes in flowering pathways have been reported to lie in this region. These results indicate that the DRM1 is very likely a novel flowering promoting locus. However, a final conclusion cannot be made until the detection of the candidate gene and the completion of transgenic complementation.

Diverse flowering pathways have been elucidated through characterizing groups of distinctive mutants and cloning the related genes. The autonomous flowering pathway is defined by those mutants that flower late under both long-day and short-day conditions and by their responsiveness to vernalization [5]. Molecularly, they all contain much higher levels of FLC transcript than the wildtype plant or late-flowering mutants associated with the photoperiod flowering pathway or the GA pathway [13, $19,20]$. Our results of vernalization and photoperiod treatments, as well as part result of the RT-PCR, strongly suggest that the $d r m 1$ is a typical late flowering mutant and most likely associated with the autonomous pathway. The conclusion is further confirmed by the finding that the transcript level of $F L C$ gene is constantly up-regulated while those of SOC1 (AGL20), FT and LEY genes are down-regulated in the $d r m 1$. In addition, no detection of significant differences in the transcript levels of GI, CO, $S P Y, F C A$ and $L D$ helps to exclude other possible pathways associated. Elucidating the mechanism of how the $D R M 1$ works together with other known components in the autonomous flowering pathway is under way. 
Supple Tab. 1 Gene-specific primers used for flowering time genes

\begin{tabular}{|c|c|c|c|}
\hline $\begin{array}{l}\text { Gene } \\
\text { name }\end{array}$ & Accession & $\begin{array}{l}\text { Oligonucleotide sequences } \\
\qquad 5^{\prime} \longrightarrow 3^{\prime}\end{array}$ & $\begin{array}{c}\text { Amplification } \\
\text { cycles }\end{array}$ \\
\hline \multirow{2}{*}{ SOC1 } & \multirow{2}{*}{ AY007726 } & F: CGAGCAAGAAAGACTCAAGTGTTTAAGG & \\
\hline & & R: GAAGTGACTGAGAGAGAGAGAGTGAG & 25 \\
\hline \multirow{2}{*}{$\mathrm{CO}$} & \multirow{2}{*}{ AY086574 } & F: AGGTGATAAGGATGCCAAGG & \\
\hline & & R: CAGGGTCAGGTTGTTGCTCT & 40 \\
\hline \multirow{2}{*}{$E M F 1$} & \multirow{2}{*}{ AF319968 } & F: TAGCAACGCAAGGAGGGAA & \\
\hline & & R: CGGGAAGGCTCTTGTGTGA & 30 \\
\hline \multirow{2}{*}{$E M F 2$} & \multirow{2}{*}{ AB053171 } & F: GGCAAGACTCGTTTCTCCTAAGC & \\
\hline & & R: GCAACAAGGAAGAGGAAGGATGT & 32 \\
\hline \multirow{2}{*}{$F C A$} & \multirow{2}{*}{ Z82990 } & F: CAGCCATTGATTGTTCGG & \\
\hline & & R: AAATTAGTTTGCGGACGGAG & 30 \\
\hline \multirow{2}{*}{$F L C$} & \multirow{2}{*}{ AF116527 } & F: CCCCATATGGGAAGAAAA AAACTAG & \\
\hline & & R: CCCGGATCCCTAATTAAGTAGTGGGAG & 35 \\
\hline \multirow[t]{2}{*}{$F T$} & \multirow[t]{2}{*}{ AF152096 } & F: ACTATATAGGCATCATCACCGTTCGTTACTCG & \\
\hline & & F: ACTATATAGGCATCATCACCGTTCGTTACTCG & 35 \\
\hline \multirow{2}{*}{$G I$} & \multirow{2}{*}{ AF105064 } & F: AACCATCTTCTGTGGGGACT & \\
\hline & & R: AGAACCCTGCGAGTCTATCA & 30 \\
\hline \multirow{2}{*}{$L D$} & \multirow{2}{*}{ U03456 } & F: ATGGAACTTGACGAACTCTGGA & \\
\hline & & R: GGGTTTTCCCGAAGTTAGTG & 30 \\
\hline \multirow{2}{*}{$L F Y$} & \multirow{2}{*}{ M91208 } & F:GCTAAAGACCGTGGCGAA & \\
\hline & & R:GCATCCACCACGTCCAGA & 34 \\
\hline \multirow{2}{*}{$S P Y$} & \multirow{2}{*}{ U62135 } & F: GCTTCACAAGATTACACCCTC & \\
\hline & & R: CGTAGTATTTGGATAGCCGA & 35 \\
\hline \multirow{2}{*}{ TFL1 } & \multirow{2}{*}{ U77674 } & F: ATGGAGAATATGGGAACTAGAG & \\
\hline & & R: TGGAACATCTGGGTCTATCATC & 35 \\
\hline \multirow{2}{*}{ ACTIN11 } & \multirow{2}{*}{ U27981 } & F: GATTTGGCATCACACTTTCTACAATG & \\
\hline & & R: GTTCCACCACTGAGCACAATG & 21 \\
\hline
\end{tabular}

The phenotypic severity of late flowering as well as vegetative growth and floral development in the $\mathrm{drm} 1$ is likely associated with the coordinate action of three repressors, FLC, EMF and TFL1

As indicated previously, $F L C$ is a key repressor component involved in both the autonomous pathway and the vernerization pathway. $E M F$ down regulates most of the flower organ genes and thus probably acts via global repression of the flower program rather than on a single flowering or flower organ identity gene [29]. TFL1 functions to suppress flower formation at the apex and to delay the transition from vegetative to reproductive development $[1,36,41]$. It has been proposed that TFL 1 acts by influencing a central mechanism controlling the identity of shoot apical meristem and consequently by preventing the expression of floral meristem identity genes, such as $A P 1$, $L F Y$ and $C A L$ at all stages of development throughout the life cycle $[35,36]$. The over-expression of TFL1 greatly extends the vegetative and inflorescence growth phases, resulting in more highly branched plants which form flowers much late than the wild type $[36,41]$.

It has been demonstrated that FLC, EMF and TFL1 function in parallel pathways to regulate floral develop- ment and subsequent flowering process $[29,36]$. In our study, it was showed that the transcript levels of all the three important repressors were constantly up-regulated in the $d r m 1$ at all developmental phases, except for that of $F L C$ in 20 d-old seedlings. It is probably due to some kind of inactivation of the FLC gene in such an early stage seedlings harvested for RNA extraction. These findings suggest that the DRM1 is likely a flowering activator through suppressing the repressors to control flowering time and to regulate the development of architectures of inflorescence and flower. The coordinate action of the three repressors in a negatively additive way helps to explain the much more severe phenotype of the drm 1 than those of other related late flowering mutants. However, more genetic evidence is needed to establish the exact relationship of the DRM1 to these repressors as well as to other components of the autonomous flowering pathway.

\section{ACKNOWLEDGMENTS}

The work was supported by a National Science Foundation of China grant (39870452) to Ben Ke Kuai. Mutant screening was conducted at Dong Lab, DCMB group, Department of Biology, Duke University. We are grateful 
to Profs. Dong $\mathrm{X}$ and Huang $\mathrm{H}$ for their valuable advices and help. We would also like to thank Zhang Q, Zhang W and Sung WJ for their technical assistances.

\section{Received, Aug 4, 2004}

Revised, Nov 20, 2004

Accepted, Dec 22, 2004

\section{REFERENCES}

1 Levy YY, Dean C. Control of flowering time. Curr Opin Plant Biol 1998; 1:49-54.

2 Mouradov A, Cremer F, Coupland G. Control of flowering time: interacting pathways as a basis for diversity. Plant Cell 2002; 14 Suppl:S111-30.

3 Simpson GG, Dean C. Arabidopsis, the Rosetta stone of flowering time? Science 2002; 296:285-9.

4 Boss PK, Bastow RM, Mylne JS, Dean C. Multiple Pathways in the Decision to Flower: Enabling, Promoting, and Resetting. Plant Cell 2004; 16:S18-31.

5 Koornneef M, Hanhart CJ, van der Veen JH. A genetic and physiological analysis of late flowering mutants in Arabidopsis thaliana. Mol Gen Genet 1991; 229:57-66.

6 Doyle MR, Davis SJ, Bastow RM, et al. The ELF4 gene controls circadian rhythms and flowering time in Arabidopsis thaliana. Nature 2002; 419:74-7.

7 Hayama R, Coupland G. Shedding light on the circadian clock and the photoperiodic control of flowering. Curr Opin Plant Biol 2003; 6:13-9.

8 Lin C. Plant blue-light receptors. Trends Plant Sci 2000; 5:337-42.

9 Mockler T, Yang H, Yu X, et al. Regulation of photoperiodic flowering by Arabidopsis photoreceptors. Proc Natl Acad Sci U S A 2003; 100:2140-5.

10 Schaffer R, Ramsay N, Samach A, et al. The late elongated hypocotyl mutation of Arabidopsis disrupts circadian rhythms and the photoperiodic control of flowering. Cell 1998; 93:1219-29.

11 Wang ZY, Tobin EM. Constitutive expression of the CIRCADIAN CLOCK ASSOCIATED 1 (CCA1) gene disrupts circadian rhythms and suppresses its own expression.Cell 1998; 93: 1207-17.

12 Olszewski N, Sun TP, Gubler F. Gibberellin signaling: biosynthesis, catabolism, and response pathways. Plant Cell 2002; 14 Suppl:S61-80.

13 Michaels SD, Amasino RM. FLOWERING LOCUS C encodes a novel MADS domain protein that acts as a repressor of flowering. Plant Cell 1999; 11:949-56.

14 Michaels SD, Amasino RM. Loss of FLOWERING LOCUS C activity eliminates the late-flowering phenotype of FRIGIDA and autonomous pathway mutations but not responsiveness to vernalization. Plant Cell 2001; 13:935-41.

15 Gendall AR, Levy YY, Wilson A, Dean C. The VERNALIZATION 2 gene mediates the epigenetic regulation of vernalization in Arabidopsis. Cell 2001; 107:525-35.

16 Chandler J, Wilson A, Dean C. Arabidopsis mutants showing an altered response to vernalization. Plant J 1996; 10:637-44.

17 Lee H, Xiong L, Gong Z, et al. The Arabidopsis HOS1 gene negatively regulates cold signal transduction and encodes a RING finger protein that displays cold-regulated nucleo-cytoplasmic partitioning. Genes Dev 2001; 15:912-24.

18 Zhang H, van Nocker S. The VERNALIZATION INDEPENDENCE 4 gene encodes a novel regulator of FLOWERING LOCUS C. Plant J 2002; 31:663-73.
19 Sheldon CC, Burn JE, Perez PP, et al. The FLF MADS box gene: a repressor of flowering in Arabidopsis regulated by vernalization and methylation. Plant Cell 1999; 11:445-58.

20 Sheldon CC, Finnegan EJ, Rouse DT, et al.The control of flowering by vernalization. Curr Opin Plant Biol 2000; 3:418-22.

21 Kobayashi Y, Kaya H, Goto K, Iwabuchi M, Araki T. A pair of related genes with antagonistic roles in mediating flowering signals. Science 1999; 286:1960-2.

22 Blazquez MA, Weigel D. Independent regulation of flowering by phytochrome B and gibberellins in Arabidopsis. Plant Physiol 1999; 120:1025-32.

23 Lee H, Suh SS, Park E, et al. The AGAMOUS-LIKE 20 MADS domain protein integrates floral inductive pathways in Arabidopsis. Genes Dev 2000; 14:2366-76.

24 Blazquez MA, Weigel D. Integration of floral inductive signals in Arabidopsis. Nature 2000; 404:889-92.

25 Busch MA, Bomblies K, Weigel D. Activation of a floral homeotic gene in Arabidopsis. Science 1999; 285:585-7.

26 Krizek BA, Meyerowitz EM. The Arabidopsis homeotic genes APETALA3 and PISTILLATA are sufficient to provide the B class organ identity function. Development 1996; 122:11-22.

27 Wagner D, Sablowski RW, Meyerowitz EM. Transcriptional activation of APETALA1 by LEAFY. Science 1999; 285:582-4.

28 Noh YS, Bizzell CM, Noh B, Schomburg FM, Amasino RM. EARLY FLOWERING 5 acts as a floral repressor in Arabidopsis. Plant J 2004; 38:664-72.

29 Sung ZR, Chen L, Moon YH, Lertpiriyapong K. Mechanisms of floral repression in Arabidopsis. Curr Opin Plant Biol 2003; 6: 29-35.

30 Hartmann U, Hohmann S, Nettesheim K, et al. Molecular cloning of SVP: a negative regulator of the floral transition in Arabidopsis. Plant J 2000; 21:351-60.

31 Aukerman MJ, Sakai H. Regulation of flowering time and floral organ identity by a MicroRNA and its APETALA2-like target genes. Plant Cell 2003; 15:2730-41.

32 Reeves PH, Murtas G, Dash S, Coupland G. early in short days 4, a mutation in Arabidopsis that causes early flowering and reduces the mRNA abundance of the floral repressor FLC. Development 2002; 129:5349-61.

33 Scortecci K, Michaels SD, Amasino RM. Genetic interactions between FLM and other flowering-time genes in Arabidopsis thaliana. Plant Mol Biol 2003; 52:915-22.

34 Chen L, Cheng JC, Castle L, Sung ZR. EMF genes regulate Arabidopsis inflorescence development. Plant Cell 1997; 9: 201124.

35 Bradley D, Ratcliffe O, Vincent C, Carpenter R, Coen E. Inflorescence commitment and architecture in Arabidopsis. Science 1997; 275:80-3.

36 Ratcliffe OJ, Amaya I, Vincent CA, et al. A common mechanism controls the life cycle and architecture of plants. Development 1998; 125:1609-15.

37 Bell CJ, Ecker JR. Assignment of 30 microsatellite loci to the linkage map of Arabidopsis. Genomics 1994; 19:137-44.

38 Lukowitz W, Gillmor CS, Scheible WR. Positional cloning in Arabidopsis. Why it feels good to have a genome initiative working for you. Plant Physiol 2000; 123:795-805.

39 Jander G, Norris SR, Rounsley SD, et al. Arabidopsis map-based cloning in the post-genome era. Plant Physiol 2002; 129:440-50.

40 Kenyon C. A conserved regulatory system for aging. Cell 2001; 105: $165-8$.

41 Ratcliffe OJ, Bradley DJ, Coen ES. Separation of shoot and floral identity in Arabidopsis. Development 1999; 126:1109-20. 